\title{
Curves in $\mathbb{Z}^{n}$
}

\author{
Grit Thürmer* \\ Computer Graphics, Visualization, Man-Machine Communication Group \\ Faculty of Media \\ Bauhaus-University Weimar \\ 99421 Weimar, Germany \\ thuermer@medien.uni-weimar.de
}

\begin{abstract}
A new definition of closed curves in $n$-dimensional discrete space is proposed. This definition can be viewed as a generalization of closed quasi curves and is intended to overcome the limitations of known definitions for practical purposes. Following the proposed definition, a set of points forms a closed curve in discrete space if the set admits a parameterization, i.e. there exists a Hamiltonian cycle in the set. Adjacencies that do not indicate the parameterization are allowed only between points that are "close to each other" along the parameterization. Additionally, it is proven that discrete curves satisfying the new definition in two-dimensional discrete space have the Jordan property.
\end{abstract}

\section{Introduction}

Curves are basic objects in geometry and play an important role in modeling. New application fields of volume data have been emerging during the last years, which require modeling of synthetic discrete objects, i.e. geometrically defined objects in $Z^{3}$ or data sets generated by rastering [1]16] geometric descriptions of objects given in $\mathbb{R}^{3}$. Such application fields are, e.g., virtual reality in medicine [17], volume-based interactive design and sculpturing [1514.

Curves in two-dimensional discrete space, i.e. in $\mathbb{Z}^{2}$, are a well explored subject 1274]. The certainly best known definition is the one of simple closed curves [10:11]. This definition is very restrictive for many practical purposes. To overcome this problem, closed quasi curves have been introduced 7]. Although, the definition of closed quasi curves works well in many cases in $\mathbb{Z}^{2}$, it is still rather limited in higher dimensional space. This point is further discussed in Sect. 4.

In this paper, a new definition of closed quasi curves is proposed. This definition can be viewed as a generalization of quasi curves as introduced in [7] and overcomes the limitations of them. Moreover, the definition is given for curves in $n$-dimensional discrete space.

The paper is organized as follows: Section 2 gives some definitions and notations used throughout the paper. In Sect. 3 simple closed curves are explored,

\footnotetext{
* This work has been supported by a postdoctoral grant of the DFG (Deutsche Forschungsgemeinschaft).
}

A. Braquelaire, J.-O. Lachaud, and A. Vialard (Eds.): DGCI 2002, LNCS 2301, pp. 34 44 2002.

(C) Springer-Verlag Berlin Heidelberg 2002 
and Sect. 4 deals with closed quasi curves. Afterwards, the new definition of closed quasi curves is suggested and discussed in Sect. 5. Finally, Sect. 6] summarizes the paper.

\section{Definitions and Notations}

The $n$-dimensional discrete space $\mathbb{Z}^{n}$ is constituted by the $n$-dimensional array of points with integer coordinates in the Cartesian coordinate system. A few definitions are given below particularly regarding $\mathbb{Z}^{2}$ and $\mathbb{Z}^{3}$. These definitions can be easily modified and extended to a discrete space of higher dimension.

Adjacency relations. Two points $p\left(x_{p}, y_{p}\right)$ and $q\left(x_{q}, y_{q}\right)$ of $Z^{2}$, where (| $\left.x_{p}-x_{q} \mid \leq 1\right) \wedge\left(\left|y_{p}-y_{q}\right| \leq 1\right)$, are said to be 4-adjacent if $\left|x_{p}-x_{q}\right|+\mid$ $y_{p}-y_{q} \mid=1$, and 8-adjacent if $0<\left|x_{p}-x_{q}\right|+\left|y_{p}-y_{q}\right| \leq 2$.

Two points $p\left(x_{p}, y_{p}, z_{p}\right)$ and $q\left(x_{q}, y_{q}, z_{q}\right)$ of $Z^{3}$, where $\left(\left|x_{p}-x_{q}\right| \leq 1\right) \wedge(\mid$ $\left.y_{p}-y_{q} \mid \leq 1\right) \wedge\left(\left|z_{p}-z_{q}\right| \leq 1\right)$, are said to be 6-adjacent if $\left|x_{p}-x_{q}\right|+\left|y_{p}-y_{q}\right|$ $+\left|z_{p}-z_{q}\right|=1$, 18-adjacent if $0<\left|x_{p}-x_{q}\right|+\left|y_{p}-y_{q}\right|+\left|z_{p}-z_{q}\right| \leq 2$, and 26-adjacent if $0<\left|x_{p}-x_{q}\right|+\left|y_{p}-y_{q}\right|+\left|z_{p}-z_{q}\right| \leq 3$.

Points $k$-adjacent to $p$, where $k \in\{4,8,6,18,26\}$, are called $k$-neighbours of $p$. The points in the $k$-neighbourhood of a point $p$ are the points $k$-adjacent to $p$ and $p$ itself, and are denoted with $N_{k}(p)$.

In $Z^{n}, n$ different types of adjacency relations can be defined, similarly as stated above for $n=2,3$. Following the definitions of adjacency relations as introduced for $Z^{2}$ and $Z^{3}$, one type of adjacency relation is a subset of the other. For example, in $\mathbb{Z}^{3}$, the set of 6 -adjacencies is a subset of the set of 18 -adjacencies, which in turn is a subset of the set of 26 -adjacencies. This correlation of the types of adjacency relation can be used as criterion for an ordering, such that for a point $p$ in $Z^{n}$ the adjacency relation of the $s^{t h}$-order with $0<s \leq n$ covers all neighbouring points of $\mathbb{Z}^{n}$ that vary in exactly $s$ coordinates from $p$.

Paths. A $k$-path $P$ in $\mathbb{Z}^{n}$ is a sequence of distinct points $P=\left\{p_{0}, p_{1}, \ldots, p_{h}\right\}$ of $Z^{n}$ such that any two consecutive points $p_{i}$ and $p_{i+1}$ with $0 \leq i<h$ along the sequence are $k$-adjacent. The $k$-path $P$ is said to be a closed path if $p_{0}$ is $k$-adjacent to $p_{h}$.

Connectivity. Two points $p, q$ are said to be $k$-connected in a subset $A$ of $\mathbb{Z}^{n}$ if there exists a $k$-path from $p$ to $q$ consisting entirely of points of $A$. A set $A$ of $\mathbb{Z}^{n}$ is said to be $k$-connected if every two points of $A$ are $k$-connected in $A$. A component $A$ is $k$-adjacent to a point $p$, and equivalently $p$ is $k$-adjacent to $A$, if $A$ contains some $k$-neighbour of $p$. The $t^{\text {th }}$-order of connectivity is defined by the types of adjacency of $s^{t h}$-order with $0<s \leq t$. For example, a set in $Z^{3}$ connected in the $2^{n d}$-order is 18 -connected. 
Graph-theoretical approach. Considering $\mathbb{Z}^{n}$ as the set of vertices $V$ of a non-oriented graph $G(V, A)$ and the adjacencies between the points of $Z^{n}$ as set of edges $A$ is commonly defined as graph-theoretical approach [2] and the graph $G$ itself is referred to as adjacency graph. An object $O \subset \mathbb{Z}^{n}$ then becomes a subgraph $G^{\prime}\left(V^{\prime}, A^{\prime}\right)$ of $G$ with $O=V^{\prime} \subset V$ and $A^{\prime} \subset A$ where $A^{\prime}$ is the set of edges representing the adjacencies between the points of $O$.

\section{Simple Closed Curves}

A curve in continuous space $\mathbb{R}^{n}$ is denoted with $\gamma$ and, in contrast, a curve in $\mathbb{Z}^{n}$ is denoted with $\gamma_{d}$ throughout the paper.

In continuous space, the Jordan Curve Theorem considers a closed curve $\gamma$ homeomorphic to a circle in $\mathbb{R}^{2}[9]$ and states that such a curve $\gamma$ separates $\mathbb{R}^{2}$ into two disjoint connected sets. This feature of $\gamma$ is known as Jordan property, which is an important property of closed curves in $\mathbb{R}^{2}$ and shall be kept for closed curves in discrete space. The Jordan curve theorem was originally proposed for discrete curves in $Z^{2}$ by ROSENFELD [1011 for pairs of adjacency relations $(k, l)$ where $k$ represents the connectedness of a curve $\gamma_{d}$ and $l$ represents the connectedness of $\mathbb{Z}^{2}-\gamma_{d}$. The Jordan curve theorem holds for $(8,4)$ and $(4,8)$.

Theorem 1 (Discrete Jordan Curve Theorem [2]). Let $\gamma_{d}$ be an $k$ connected set of $\mathbb{Z}^{2}$ containing at least five points and any point $p$ of $\gamma_{d}$ is $k$-adjacent to exactly two other points of $\gamma_{d}$. Then $\mathbb{Z}^{2}-\gamma_{d}$ has exactly two l-components, each of which is l-connected to every point of $\gamma_{d}$.

A curve for which Theorem 1 holds is called a simple curve. Since a Jordan curve in $\mathbb{R}^{2}$ is a one-manifold, it is possible to derive an analogous definition of "one-manifold" discrete closed curves from the theorem. These are finite curves in $Z^{2}$ for which the theorem holds:

Definition 1 (Simple Closed Curve in $\mathbb{Z}^{2}[\mathbf{1 3}]$ ). $A$ simple closed curve $\gamma_{d}$ is a finite set of points in $\mathbb{Z}^{2}$ for which the following conditions hold.

SCC1 $\gamma_{d}$ is k-connected.

SCC2 $\gamma_{d}$ consists of at least five points.

SCC3 Each point of $\gamma_{d}$ is $k$-adjacent to exactly two other points of $\gamma_{d}$.

Condition SCC2 was intended to rule out cases for which $Z^{2}-\gamma_{d}$ is not separated into two non-empty subsets as illustrated in Fig. 1(a) for a 4-connected curve, and in (b) for an 8-connected curve. Note, that this condition is actually too restrictive for 8-connected curves. An example is given in Fig. 11 c) for which an 8-connected curve separates $\mathbb{Z}^{2}$ into two components and consists itself of four points. Using the terminology of the graph-theoretical approach, a simple closed curve $\gamma_{d}$ is a connected graph in which any vertex has degree two.

An arc is defined as an $k$-connected set of points, denoted with $\alpha$, with each of its points satisfying the conditions SCC1-3, except two points which are $k$ adjacent to just one other point of $\alpha$. In 13, ROSENFELD has shown that no simple closed curve in $\mathbb{Z}^{2}$ is both a 4 -connected curve and an 8-connected curve. 


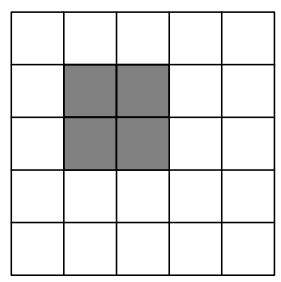

(a)

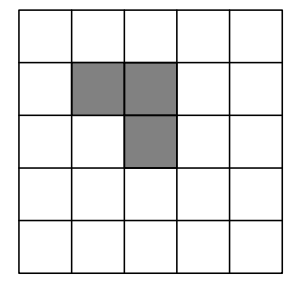

(b)

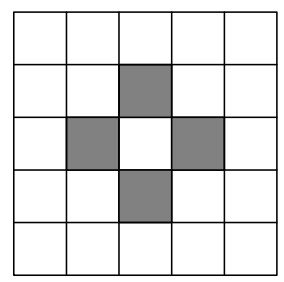

(c)

Fig. 1. Simple closed curves: (a) a 4-connected set to be ruled out, (b) an 8-connected set to be ruled out, (c) a valid 8-connected curve.

The generalization of Def. 1 to simple closed curves in $Z^{n}$ is straightforward. Subsequently, we talk about simple curves if we do not care about closeness of $\gamma_{d}$, i.e. a simple curve is a closed curve or it is an infinite set of points, for which SCC1-3 are true.

\section{Closed Quasi Curves}

Often, a discrete curve $\gamma_{d}$ is the result of a rasterization of a continuous curve $\gamma$ or it is the border of a two-dimensional object rastered in $Z^{2}$. The generation of a simple curve $\gamma_{d}$ as a discrete representation of a continuous curve $\gamma$, that possesses high curvature, is a well-known problem. It has been shown in [5], that a simple curve cannot be obtained generally even in areas of $\gamma$ with almost no curvature, unless $\gamma$ is a straight line. This may necessitate an elimination of "additional" points of the rasterization to obtain a simple curve. That problem in discrete space has been already reported, e.g., in 7. An example is given in Fig. 2(a) for a rastered circle. The points in light grey would have been eliminated to obtain a simple closed 8-connected curve in $\mathbb{Z}^{2}$.

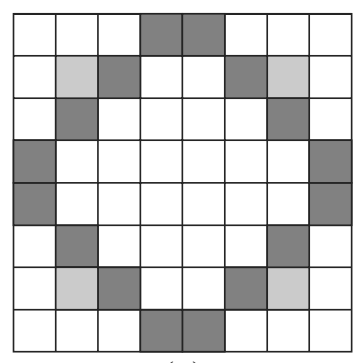

(a)

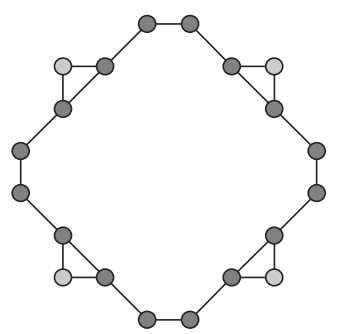

(b)

Fig. 2. (a) 8-connected closed quasi curve, (b) adjacency graph. 
As shown above, the definition of simple curves is too restrictive for many cases. To overcome the problem, a generalization of simple closed curves has been proposed by MALGOUYRES in [7, which were named closed quasi curves. Closed quasi curves admit - like simple closed curves - a unique parameterization, that has been identified as one of the fundamental features of curves to be kept in discrete space. Loosely speaking, a closed quasi curve is obtained by adding "triangles" to the adjacency graph of simple closed curves following certain rules. This is illustrated for an example in Fig. 2(b). The notation of closed quasi curves are generally stated for graphs in 8] and for 18-connected closed quasi curves in [7. The latter one is given below and can be easily adapted to 8-connected curves in $\mathbb{Z}^{2}$.

Definition 2 (18-connected Closed Quasi Curve). A set $\gamma_{d}$ of $\mathbb{Z}^{3}$ is said to be an 18-connected closed quasi curve iff the cardinality of $\gamma_{d}$ is greater or equal to four and $\gamma_{d}$ satisfies the following properties.

CQC1 $\gamma_{d}$ is 18-connected.

CQC2 Any point of $\gamma_{d}$ is 18-adjacent to either two, three or four points of $\gamma_{d}$.

CQC3 For any subset $\alpha$ of $\gamma_{d}$ consisting of three points such that the elements

of $\alpha$ are pairwise 18-adjacent, there is a point $p \in \alpha$ which is 18-adjacent to no point in $\gamma_{d}-\alpha$. If $q$ and $r$ are the two points of $\alpha-p$, then $q$ and $r$ satisfy the following conditions.

CQC3a $q$ and $r$ are 18-adjacent to a point in $\gamma_{d}-\alpha$.

CQC3b No point in $\gamma_{d}-\alpha$ is 18-adjacent to both $q$ and $r$.

CQC4 For each point $p$ in $\gamma_{d}$ which is 18-adjacent to at least three points of $\gamma_{d}$, there exists a non-trivial partition of $N_{18}(p) \cap \gamma_{d}$ into two non-empty subsets $\alpha$ and $\beta$ such that the following conditions hold.

CQC4a The points of $\alpha$ are pairwise 18-adjacent.

CQC4b The points of $\beta$ are pairwise 18-adjacent.

CQC4c No point of $\alpha$ is 8-adjacent to a point of $\beta$.

It has been shown [6], that an 8-connected closed quasi curve $\gamma_{d}$ in $Z^{2}$ satisfies the Jordan property, i.e. $Z^{2}-\gamma_{d}$ consists of exactly two 4-connected components.

The definition of closed quasi curves as given above works well for many cases in $Z^{2}$ but is still rather limited in higher dimensional space, i.e. $Z^{3}$ as shown in Fig. 3. This example does not satisfy the Def. 2 as can be seen from the 18adjacency graph in Fig. 3(b). Either the point $q$ or $p$ must be deleted such that Def. 2 holds. Even more, considering the same curve as a 26 -connected curve, the points $q$ and $p$ and the two points adjacent to them are mutually 26-adjacent as shown in the 26-adjacency graph in Fig. 3(c). The points $p$ and $q$ can be viewed as "additional" points of a simple closed 26-connected curve, which can arise only in dimension three or higher. The point $r$ is covered by the Def. 2 as some "additional" point to simple closed curves. Consequently, we need some further generalization of closed curves. 


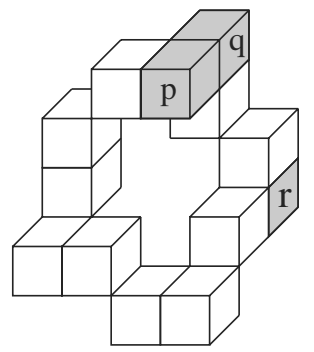

(a)

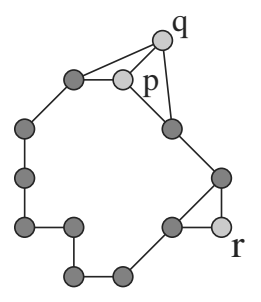

(b)

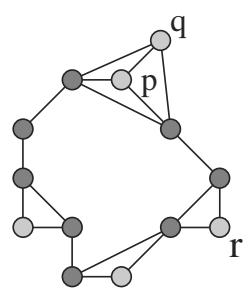

(c)

Fig. 3. (a) Closed curve in $\mathbb{Z}^{3}$, (b) 18-adjacency graph, (b) 26-adjacency graph.

\section{A New Definition of Closed Quasi Curves in $\mathbb{Z}^{n}$}

\subsection{Properties of Closed Quasi Curves}

Before we come to the new definition of closed quasi curves in $Z^{n}$, basic properties of closed curves are identified, which we wish to ensure by our definition of a discrete curve $\gamma_{d}$ in $Z^{n}$.

The parameterization is a natural and fundamental property of continuous manifold curves and, of course, must be kept for the representation of such curves in $\mathbb{Z}^{n}$. The necessity of a unique Hamiltonian cycle in the graph of a curve - and with it a unique parameterization of the curve - is very restrictive, particularly in three or higher dimensional discrete space. This is illustrated in the example of Fig. 3. On the other hand, a curve connected in the $1^{\text {st }}$-order meets our understanding of a maximal connected curve, i.e. whenever a point $p$ is added to a simple closed curve connected in the $1^{\text {st }}$-order, e.g. a 4-connected curve $\gamma_{d}$ in $Z^{2}$, the set $\gamma_{d} \cup p$ does not corresponds to the common understanding of a curve anymore. Hence, the Hamiltonian cycle in the graph of a curve should contain all adjacency relations of the $1^{\text {st }}$-order.

Fig. 4 illustrates a further problem. In both cases, (a) and (b), a Hamiltonian cycle exists (thick lines) which is not unique. The point $r$ can be viewed as some "additional" point with respect to a simple closed curve. However, in (a) the adjacency between the points $r$ and $q$ violates the understanding of a curve since the neighbours $p$ and $q$ of $r$ have a common neighbour which is not adjacent to $r$. Hence, in Fig. 4(a) $r$ is not a valid "additional" point of a curve, but in (b) it is. Consequently, a specification of the adjacencies, that do not indicate the parameterization, is required.

\subsection{Definition of Closed Quasi Curves}

Assume the graph $G\left(\gamma_{d}, B\right)$ of a $k$-connected curve $\gamma_{d}$ where $B$ are the edges representing the $k$-adjacencies between the points of $\gamma_{d}$. The properties of closed curves as discussed in the previous section are kept using the following definition. 


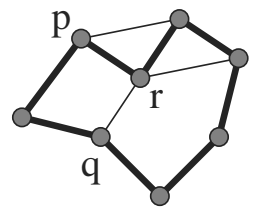

(a)

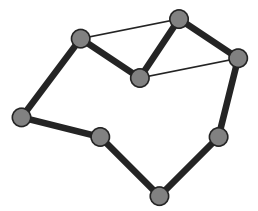

(b)

Fig. 4. Hamiltonian cycle in a closed curve (a) invalid (b) valid.

Definition 3 (Closed Quasi Curve in $\mathbb{Z}^{n}$ ). A k-connected subset $\gamma_{d}$ of $\mathbb{Z}^{n}$ is a closed quasi curve in $\mathbb{Z}^{n}$ iff $\gamma_{d}$ satisfies the following properties.

CQC1' There exists a Hamiltonian cycle $H\left(\gamma_{d}, A\right)$ in the graph $G\left(\gamma_{d}, B\right)$, where $A \subset B$ and $A$ contains all edges representing the adjacencies of the $1^{\text {st }}$-order between points of $\gamma_{d}$.

CQC2' For each point $p$ of $\gamma_{d}$ the subgraph $H^{\prime}\left(N_{k}(p) \cap \gamma_{d}, A^{\prime}\right)$ of $H\left(\gamma_{d}, A\right)$ induced by the points of $\gamma_{d}$ in the $k$-neighbourhood of $p$, constitutes a $k$-path. CQC3' If for $H\left(\gamma_{d}, A\right)$ A represents exclusively adjacencies of the $1^{\text {st }}$-order, the cardinality of $\gamma_{d}$ is greater or equal to five and it is greater or equal to four, otherwise.

Condition CQC1' can be verified as follows. Associate each element of $B$ of the graph $G$ with the order of the adjacency represented. Then the problem of finding any Hamiltonian cycle in $G$ is replaced by finding a Hamiltonian cycle $H$ of minimal length in the weighted graph $G$. This is a special case of the traveling salesman problem [3]: each pair of vertices with an edge between them has a distance depending on the weight, while pairs of vertices without an edge between them are separated by a distance of infinite length. When a Hamiltonian cycle of minimal length has been obtained in $G$, the cycle must contain all adjacencies of the $1^{\text {st }}$-order. Otherwise, the set is not a quasi curve.

Since each point of $\gamma_{d}$ has degree two in $H\left(\gamma_{d}, A\right)$ and $A$ contains all adjacencies of the $1^{\text {st }}$-order, no point of a closed quasi curve has more than two neighbours adjacent in the $1^{\text {st }}$-order.

Remark 1. If any point in the set $\gamma_{d}$ has more than two neighbours of the $1^{\text {st }}$ order of adjacency, the set is not a quasi curve.

Condition CQC2' of Def. 3 ensures that any additional adjacency with respect to a simple curve arise only between points that are "close to each other" along the parameterization. Such additional adjacencies may arise in areas where curves perform high curvature.

Finally, condition CQC3' rules out cases, e.g. as shown in Fig.11(a), in which $Z^{2}-\gamma_{d}$ is not separated.

In many cases a closed quasi curve connected in the $1^{\text {st }}$-order is also a closed quasi curve connected in some higher order. This fact is illustrated by examples in Fig. [5(a) and (c) for a curve in $Z^{2}$ and $Z^{3}$, respectively. The thick lines in the adjacency graphs represent the adjacencies of the $1^{s t}$-order. Note that the 
example in Fig. 5(a) is not an 8-connected closed quasi curve according to the definition by MALGOUYRES.

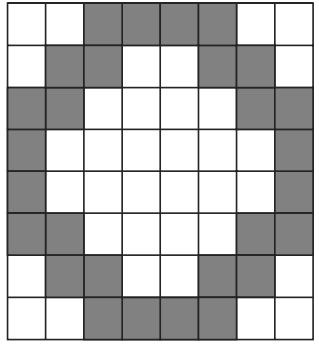

(a)

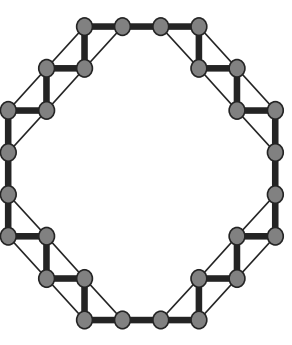

(b)

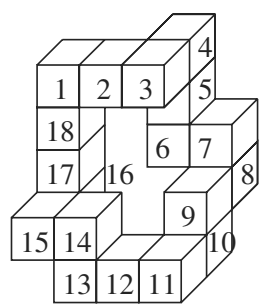

(c)

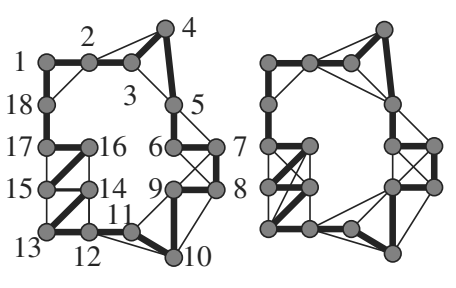

(d)

(e)

Fig. 5. (a) Closed quasi curve in $\mathbb{Z}^{2}$ with (b) the adjacency graph for the 8connectedness, and (c) closed quasi curve in $\mathbb{Z}^{3}$ with adjacency graph for the (d) 18-connectedness and (e) 26-connectedness.

The examples in Fig. 5] suggest that any closed quasi curve connected in the $1^{\text {st }}$-order is also a closed quasi curve connected in some higher order and, inversely, for each closed quasi curve $\gamma_{d}$ connected in an order greater than one there exists a closed quasi $\gamma_{d}^{\prime}$ connected in the $1^{\text {st }}$-order such that $\gamma_{d} \subset \gamma_{d}^{\prime}$. This reasoning is not true, as can be seen from the examples in Fig. 6. in (a) and (c) closed quasi curves connected in the $1^{\text {st }}$-order are shown that are not quasi curves for any higher order of connectivity, because condition CQC2' of Def. 3 would not be satisfied in these cases. For the example in Fig. 6(b), there does not exist any 4-connected closed quasi curve $\gamma_{d}^{\prime}$, of which the shown curve $\gamma_{d}$ is a subset. Indeed, it is impossible to identify two 4-neighbours of $p$ - call them $q$ and $r$ - which connect $p$ with the set $\gamma_{d}-p$ in the $1^{s t}$-order such that each point $q$ and $r$ has exactly two 4-neighbours in $\gamma_{d}^{\prime}$.

For a closed quasi curve that satisfies Def. 3 and is connected in the $1^{\text {st }}$-order, the following is true:

Theorem 2. A closed quasi curve $\gamma_{d}$ in $\mathbb{Z}^{n}$ connected in the $1^{\text {st }}$-order is a simple closed curve.

Proof. Assume the adjacency graph $G\left(\gamma_{d}, A_{1}\right)$ of a closed quasi curve $\gamma_{d}$ connected in the $1^{\text {st }}$-order. Then $A_{1}$ covers all adjacencies of the $1^{\text {st }}$-order between 


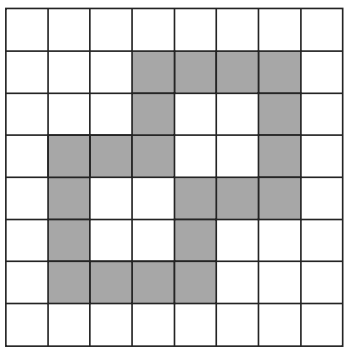

(a)

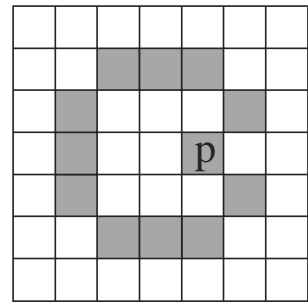

(b)

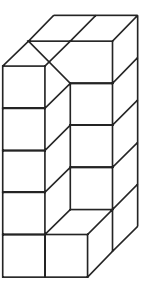

(c)

Fig. 6. (a) 4-connected closed quasi curve, (b) 8-connected closed quasi curve, and (c) 6-connected closed quasi curve.

the points of $\gamma_{d}$. Since the Hamiltonian cycle $H\left(\gamma_{d}, A\right)$ contains all adjacencies of the $1^{\text {st }}$-order $H\left(\gamma_{d}, A\right)=G\left(\gamma_{d}, A_{1}\right)$. Consequently, each point of $\gamma_{d}$ is adjacent in the $1^{s t}$-order to exactly two other points of $\gamma_{d}$. Moreover, following Def. 3 the cardinality of $G$ is equal or greater to five.

From Theorem 2 follows that a 4 -connected closed quasi curves in $Z^{2}$ that satisfies Def. 3 is a 4 -connected simple closed curve. The Jordan Curve Theorem 1 is true for a simple closed curve. Consequently, the Jordan Curve Theorem is also true for a 4-connected closed quasi curves in $\mathbb{Z}^{2}$ :

Corollary 1. A 4-connected closed quasi curve $\gamma_{d}$ in $\mathbb{Z}^{2}$ separates $\mathbb{Z}^{2}-\gamma_{d}$ in exactly two 8-components each of which is 8-connected to every point of $\gamma_{d}$.

Finally, it is left to show that an 8-connected closed quasi curve in $\mathbb{Z}^{2}$ satisfying the new definition has the Jordan property. For this, the terms maximal and minimal connected closed quasi curve are used and introduced below.

A maximal connected closed quasi curve is a closed quasi curve that satisfies Def. 3 and to which no point can be added such that the result still fulfills the conditions of Def. 3. For example, a 4-connected closed quasi curve in $Z^{2}$ is a maximal connected curve. As stated in Theorem 2 a 4-connected closed quasi curve $\gamma_{d}$ in $Z^{2}$ is a simple closed curve. Thus, each point of $\gamma_{d}$ has exactly two 4-neighbours. Adding a point $p$ to $\gamma_{d}$ such that $p$ is 4-connected to $\gamma_{d}$ leads to at least one point of $\gamma_{d}$ that has more than two 4-neighbours in $\gamma_{d} \cup p$. As already stated in Remark 1 then the set $\gamma_{d} \cup p$ is not a closed quasi curve.

In contrast, a minimal connected closed quasi curve is a closed quasi curve that satisfies Def. 3 and from which no point can be deleted such that the result still fulfills the conditions of Def. 3. A minimal 8-connected closed quasi curve in $\mathbb{Z}^{2}$, which satisfies Def. 3 is a curve $\gamma_{d}$ for which the Hamiltonian cycle $H\left(\gamma_{d}, A\right)=G\left(\gamma_{d}, B\right)$, i.e. all 8-adjacencies between the points of $\gamma_{d}$ belong to the Hamiltonian cycle. In this case, each point of $\gamma_{d}$ has exactly two 8-neighbours in $\gamma_{d}$. Thus, a minimal 8-connected closed quasi curve in $\mathbb{Z}^{2}$ is an 8-connected simple closed curve. 
Theorem 3. An 8-connected closed quasi curve $\gamma_{d}$ in $Z^{2}$ that satisfies Def. 3 separates $\mathbb{Z}^{2}-\gamma_{d}$ in exactly two 4-components.

Proof. Assume $\gamma_{d}$ is a minimal connected closed quasi curve, i.e. $\gamma_{d}$ is an 8connected simple closed curve. Then $\gamma_{d}$ separates $\mathbb{Z}^{2}-\gamma_{d}$ into two 4-components.

One can view any 8-connected closed quasi curve as a minimal 8-connected closed quasi curve with added points. It will be shown below, that any point added to $\gamma_{d}$ neither removes one of the 4-components separated by the minimal curve nor increases the number of 4-components by subdividing an existing 4component.

Assume an added point $p$ removes one of the 4-components of $Z^{2}-\gamma_{d}$ and call this component $I$. Then $I$ must consist of exactly one point $q$ and all points in $N_{4}(q)-q$ belong to $\gamma_{d}$. Consequently, $p$ would have four 4-neighbours in $\gamma_{d}$ and this contradicts Def. 3. because in this case there does not exist a Hamiltonian cycle in $\gamma_{d} \cup p$ containing all 4-adjacency relations between the points of $\gamma_{d} \cup p$.

Assuming a point $p \in \mathbb{Z}^{2}-\gamma_{d}$, that is added to a minimal connected closed quasi curve $\gamma_{d}$, introduces at least one additional 4-component by subdividing an existing one such that $\mathbb{Z}^{2}-\gamma_{d}-p$ consists of at least one more 4-component than $\mathbb{Z}^{2}-\gamma_{d}$. To achieve this, $N_{8}(p)-\gamma_{d}$ must consist of $m$ 4-components and $N_{8}(p)-\gamma_{d}-p$ of at least $m+14$-components. Hence, there are at least two 8-components in $N_{8}(p) \cap \gamma_{d}$. Assume there exist a Hamiltonian cycle for the set $\gamma_{d} \cup p$ which contains all edges representing 4-adjacencies between the points of $\gamma_{d} \cup p$. To satisfy condition CQC2' of Def. 3 for any point $p$ added to a minimal 8-connected closed quasi curve $\gamma_{d},\left(N_{8}(p)-p\right) \cap \gamma_{d}$ must form an 8 -path. However, this contradicts the conditions of a point $p$ that subdivides an existing 4-component of $\mathbb{Z}^{2}-\gamma_{d}$.

\section{Summary}

It has been shown that simple closed curves and closed quasi curves proposed in [7] might be too restrictive for practical purposes. This has motivated the new definition of closed quasi curves introduced in this paper. The new definition can be viewed as some generalization of quasi curves as introduced in [7] and overcomes the limitations of them. Moreover, the definition is given for curves in $n$-dimensional discrete space and is, therefore, not restricted to any certain dimension of space. It has been proven that curves in $Z^{2}$, which satisfy the new definition, have the Jordan property.

It is a drawback of the new definition - in contrast to the known definition of quasi curves - that it cannot be decided locally for a point $p$ if this point belongs to a quasi curve considering only the neighbourhood configuration of the point. This is a point of future work. At the moment it can be stated only, that the existence of a path in the subgraph of the Hamiltonian cycle induced by the points of a quasi curve in the neighbourhood of each point of the quasi curve is necessary. It has to be shown, if this condition is already sufficient or if further local properties have to be identified. 
Consider the Jordan curve theorem for an 8-connected closed quasi curve $\gamma_{d}$ in $\mathbb{Z}^{2}$. It seems to be clear that each point of $\gamma_{d}$ is 8 -connected to the two separated sets of $Z^{2}-\gamma_{d}$. However, at the moment this is just a conjecture and needs further investigations to provide a formal proof. This is also a point of future work.

\section{References}

1. Cohen-Or, D., And Kaufman, A. Fundamentals of surface voxelization. Graphical Models and Image Processing 57, 6 (1995), 453-461.

2. Kong, T. Y., And Rosenfeld, A. Digital topology: a comparison of the graphbased and topological approaches. In Topology and category theory in computer science, G. M. Reed, A. W. Roscoe, and R. F. Wachter, Eds. Oxford University Press, 1991, pp. 273-289.

3. Lawler (ED.), E. L., AND Rinnooy-KAN, A. H. The Traveling Salesman problem: A guided tour of combinatorial optimization. Wiley-Interscience Series in Discrete Mathematics. John Wiley and Son, 1985.

4. LeBiedź, J. Discrete arcs and curves. Machine Graphics and Vision 9, 1/2 (2000), 25-30.

5. Lincke, C., AND Wüthrich, C. A. Morphologically closed surfaces and their digitization. submitted for publication.

6. Malgouyres, R. Phd thesis, Université d'Auvergne, Clermont-Ferrand, France, 1994.

7. Malgouyres, R. Graphs generalizing closed curves with linear construction of the Hamiltonian cyle - parameterization of discretized curves. Theoretical Computer Science 143 (1995), 189-249.

8. Malgouyres, R. A definition of surfaces of $Z^{3}$ : A new 3D discrete Jordan theorem. Theoretical Computer Science 186 (1997), 1-41.

9. Moise, E. E. Geometric topology in dimensions 2 and 3. Springer-Verlag, New York, 1977.

10. Rosenfeld, A. Connectivity in digital pictures. Journal of the ACM 17, 1 (1970), $146-160$.

11. Rosenfeld, A. Arcs and curves in digital pictures. Journal of the ACM 20, 1 (1973), 81-87.

12. Rosenfeld, A. A converse to the Jordan curve theorem for digital curves. Information and Control 29 (1975), 292-293.

13. Rosenfeld, A. Three-dimensional digital topology. Information and Control 50 (1981), 119-127.

14. Shareef, N., AND Yagel, R. Rapid previewing via volume-based solid modeling. In Proc. of Solid Modeling '95 (1995), pp. 281-292.

15. Wang, S. W., And Kaufman, A. E. Volume-sampled 3D modeling. IEEE Computer Graphics and Applications 14, 5 (1994), 26-32.

16. Wüthrich, C. A. A model for curve rasterization in n-dimensional space. Computers and Graphics 22, 2-3 (1998), 153-160.

17. Yagel, R., Stredney, D., Wiet, G. J., Schmalbrock, P., Rosenberg, L., Sessanna, D. J., And Kurzion, Y. Building a virtual environment for endoscopic sinus surgery simulation. Computers and Graphics 20, 6 (1996), 813-823. 